\title{
Clinical Data Mining Related to the Japanese Kampo Concept "Hie" (Oversensitivity to Coldness) in Men and Pre- and Postmenopausal Women
}

\author{
H. Tokunaga, ${ }^{1}$ K. Munakata, ${ }^{2}$ K. Katayama, ${ }^{3}$ R. Yamaguchi, ${ }^{3}$ S. Imoto, ${ }^{3}$ \\ S. Miyano, ${ }^{3}$ and K. Watanabe ${ }^{1,2}$ \\ ${ }^{1}$ Center for Kampo Medicine, School of Medicine, Keio University, 35 Shinanomachi, Shinjuku-ku, Tokyo 160-8582, Japan \\ ${ }^{2}$ Faculty of Environment and Information Study, Keio University, 532 Endo, Fujisawa, Kanagawa 252-0882, Japan \\ ${ }^{3}$ Laboratory of DNA Information Analysis, Human Genome Center, Institute of Medical Science, University of Tokyo, \\ 4-6-1 Shirokanedai, Minato-ku, Tokyo 108-8639, Japan
}

Correspondence should be addressed to K. Watanabe; watanabekenji@a6.keio.jp

Received 19 July 2013; Revised 5 December 2013; Accepted 29 December 2013; Published 24 February 2014

Academic Editor: Heidrun Reissenweber-Hewel

Copyright (C) $2014 \mathrm{H}$. Tokunaga et al. This is an open access article distributed under the Creative Commons Attribution License, which permits unrestricted use, distribution, and reproduction in any medium, provided the original work is properly cited.

\begin{abstract}
"Hie" is a subjective oversensitivity to cold and a condition experienced in 60\% of Japanese citizens. The condition of hie has not been documented in Western medicine. However, in Kampo medicine, hie is an important target of treatment, because it has been considered one of the sources of all kinds of diseases. This study aimed to clarify the symptoms and findings associated with hie and contribute to increased precision in hie diagnosis. During 2005-2006, data from interviews of 1691 patients during their initial visit to the Kampo Clinic of Keio University Hospital were analyzed using a classification and regression tree (CART) analysis, a data mining technique. Symptoms and findings characteristic of each group are follows as, postmenopausal women: fatigability, absence of lower abdominal pain, and absence of hot flashes of feet: women with menstruation: leg swelling, knee pain, and abdominal pain; men: insomnia, leg weakness, and absence of excess saliva. From the perspective of Kampo medicine the result suggested that the feature of hie condition in postmenopausal women, women with menstruation, and men is statistically different.
\end{abstract}

\section{Introduction}

"Hie" is a condition that interferes with daily life because a person experiences pain in the entire body or in some parts of the body due to a feeling of cold at temperatures that are not normally considered cold by most people [1]. Hie is experienced by about $60 \%$ of Japanese people [2, 3]. Most people complaining of oversensitivity to cold temperatures are women. Approximately $45 \%$ of these women were within the age range of $15-39$ years, whereas $49.6 \%$ were within the age range of 40-64.5 years [4]. In addition, men's oversensitivity to cold temperatures has been studied recently and was shown to have approximately one-sixth the frequency of the hie experienced by women [5].

Kampo medicine practitioners refer to hie as a physical condition in which the hands, the legs, and the lower back rapidly become cold because of poor blood circulation. In general, hie is believed to occur because of an abnormality or bias in the balance between heat production/transport and heat dissipation. Peripheral circulatory failure associated with autonomic imbalance is believed to be one of the major causes of hie, mainly attributable to stress and an irregular lifestyle. In addition, circulatory insufficiency is likely to occur because of anemia, hypotension, and poor muscular development. People with large amounts of subcutaneous fat are cut off from the temperature in the outside world and are likely to develop peripheral circulatory failure; in other words, such people are likely to develop hie. In addition, people with less muscle mass produce less heat and are likely to develop oversensitivity to cold temperatures. A number of other causes of cold oversensitivity have been identified, including decreased levels of estrogen and progesterone. In addition, external factors such as tight underwear, shoes, and socks are likely to cause a circulatory deficit; furthermore, intense 
temperature differences inside and outside of rooms because of air conditioner usage are likely to cause hypofunctioning of the autonomic nervous system, leading to hie [6].

Conversely, symptomatic oversensitivity to cold temperatures is present in Buerger's disease, Raynaud's phenomenon $[7,8]$, collagenosis, diabetic peripheral neuropathy [9], arteriosclerosis obliterans due to peripheral arterial circulatory insufficiency, and venous circulatory insufficiency, such as in thrombophlebitis, varicose veins, and venous thrombosis, as well as leprosy [10] and reflex sympathetic dystrophy (RSD) [11]. Hie is one of the symptoms of hypothyroidism.

Additionally, Western medicine has shown some of the causes of symptomatic hie. However, the term hie does not exist in Western medicine. Western medicine is currently the mainstream approach for treatment in Japan; in the absence of underlying disease, hie is perceived as constitutional. For some people, hie is a part of daily life, but most physicians considered it as a condition that does not require active treatment because no objective diagnostic method has yet been established [5].

However, Kampo physicians have regarded hie as an important target of medical treatment.

In Kampo medicine, the concept of disease prevention is very strong. An early deviance of the patient's condition should be treated before an obvious pathology has been developed. hie is considered as one of such early deviances.

For the treatment of hie as a disease entity, prescriptions covered by the health insurance include shimotsuto, keishi-bukuryogan, goshakusan, and kami-shoyosan [12]. This study aimed to educate nonspecialized physicians in Kampo medicine on the importance of treating hie and to increase awareness of the utility of Kampo medicine in such treatments.

Therefore, at the Kampo Clinic of Keio University Hospital, the determined therapeutic efficacy and relationship between the symptoms and Kampo medicine-based diagnostic "evidence" were analyzed using the following techniques: data mining based on patients' subjective medical information, Kampo medical diagnoses determined by the physicians, and prescription information. In addition, a system providing support for Kampo medical diagnosis and treatment has been developed for establishing guidelines for general physicians' use, in order to administer Kampo treatment appropriately and for the widespread use of Kampo medicine-based treatment. In this study, the symptoms and findings associated with hie were determined on the basis of accumulated patient data during their initial visit and were analyzed for elaborating the diagnosis of hie.

\section{Materials and Methods}

The study was conducted on 1691 patients (482 men and 1209 women) whose initial visit at the Kampo Clinic of Keio University Hospital occurred between April 2005 and March 2007. Findings and symptoms associated with oversensitivity to cold temperatures were analyzed using the data mining software, Clementine 12.2 (IBM), on the basis of 137 items including body mass index (BMI); blood pressure; Kampo medical findings (e.g., tongue, pulse, and abdominal examination); and subjective symptoms.

The patients who met any one of the following criteria were considered as hie patients: those whose entire body or part of the body displayed symptoms of hie and those who were at least "sensitive to cold" or who "easily develop frostbites." Patients with data deficiencies and those younger than 15 years old were excluded. Patients with atopic dermatitis were excluded because of their particular pathological background. A decision tree analysis (classification and regression tree: CART) was performed separately on the female and male hie patients (female: 464/744; men: 102/276; total: 566/1020), using the same software, and the factors related to hie were extracted.

In the CART algorithm, we focus on the data of a single variable and divide the patients into two exclusive groups, where the patients in one group have the values that are greater than the cutoff and the values of the patients in the other group are smaller. The variable used for patients' division is selected so that hie or non-hie patients are more enriched in each of the obtained patient groups than the group before division. We started with the all patients and applied the CART algorithm that generated a tree structure of patients' division, called decision tree, with the variables used. The tree structure can be regarded as a sequential classification of patients and we investigate the sequence of the used variables from a medical viewpoint.

We treated real numerical data such as BMI and blood pressure as categorical variables as much as we possibly could to improve their interpretability, according to the standards of academic societies. The standards are summarized as follows.

(i) BMI: the patients' BMI were classified into 3 categories on the basis of the standard criteria of the Japan Society for the Study of Obesity: thin (less than 18.5), standard (18.5 or higher but less than 25$)$, and obese (25 or higher).

(ii) Blood pressure: on the basis of the standard criteria of the Japanese Society of Hypertension, the patients were classified into 4 blood pressure categories: optimal, normal, high-normal, and high. In addition, although the academic society has no clear standard criteria stating that hie is often encountered in families with hypotension, patients with a systolic blood pressure of less than $100 \mathrm{mmHg}$ were classified into 1 category designated as having "hypotension." The patients' blood pressure was classified into 5 categories.

(iii) Frequency of urination: on the basis of the standard criteria of respective academic societies, the frequency of urination was classified into 2 categories. A frequency of more than 8 times per day was considered as "frequent daytime urination" (International Continence Society), whereas a urine frequency of once or more per night was considered as "nocturia" (Neurogenic Bladder Society). 
TABle 1: Demographic background of patients (Av. \pm SD).

\begin{tabular}{|c|c|c|c|c|}
\hline & Total $(n=1020)$ & Postmenopause $(n=235)$ & Menstruating $(n=509)$ & Male $(n=276)$ \\
\hline Age (years) & $47.3 \pm 17.1$ & $62.4 \pm 9.8$ & $36.1 \pm 9.6$ & $52.1 \pm 18.3$ \\
\hline Height $(\mathrm{cm})$ & $159.8 \pm 7.8$ & $154.2 \pm 5.7$ & $158.9 \pm 5.6$ & $168.1 \pm 6.2$ \\
\hline Weight $(\mathrm{kg})$ & $55.0 \pm 11.0$ & $51.3 \pm 9.4$ & $52.6 \pm 8.7$ & $64.5 \pm 11.1$ \\
\hline Body mass index $\left(\mathrm{kg} / \mathrm{m}^{2}\right)$ & $21.5 \pm 3.5$ & $21.6 \pm 3.6$ & $20.9 \pm 3.3$ & $22.8 \pm 3.4$ \\
\hline Systolic blood pressure (mmHg) & $116.9 \pm 19.1$ & $126.3 \pm 18.7$ & $109.0 \pm 15.7$ & $122.9 \pm 19.1$ \\
\hline Diastolic blood pressure $(\mathrm{mmHg})$ & $71.3 \pm 13.3$ & $74.8 \pm 12.7$ & $67.2 \pm 11.7$ & $76.7 \pm 14.1$ \\
\hline Percentage of hie symptom (\%) & 59.0 & 60.0 & 72.0 & 37.0 \\
\hline Percentage of frequent urination (\%) & 32.0 & 35.7 & 33.0 & 24.0 \\
\hline Percentage of constipation (\%) & 7.8 & 5.5 & 11.4 & 4.7 \\
\hline
\end{tabular}

TABLE 2: Selected Kampo terms (ICD11Beta).

\begin{tabular}{|c|c|c|}
\hline Kampo terms & ICD terms & Description \\
\hline Kyo & Deficiency pattern & $\begin{array}{l}\text { A pattern characterized by fatigue or weakness; at the onset of febrile } \\
\text { condition, characterized by cold sensitivity and tendency to sweat, floating } \\
\text { weak pulse; in case of nonfebrile condition, characterized by weak pulse, weak } \\
\text { abdominal wall. It may be explained by weak response to pathogens. }\end{array}$ \\
\hline Jitsu & Excess pattern & $\begin{array}{l}\text { A pattern characterized by (at the onset of febrile condition) severe chills with } \\
\text { no sweating, strong pulse; in case of nonfebrile condition, strong pulse, strong } \\
\text { abdominal wall. It may be explained by strong response to pathogens. }\end{array}$ \\
\hline Oketsu & Blood stasis patterns & $\begin{array}{l}\text { A pattern characterized by various menstrual disorders such as amenorrhea, } \\
\text { dysmenorrhea, and menopausal syndrome; lower abdominal fullness, } \\
\text { varicose veins, hemorrhoids, and mood swings. It may be explained by } \\
\text { impaired peripheral blood circulation or obstruction of venous return. }\end{array}$ \\
\hline Suidoku & Fluid disturbance patterns & $\begin{array}{l}\text { A pattern characterized by fluid retention or dehydration in the digestive } \\
\text { tract, body tissues, or cavities. It may be explained by abnormal distribution of } \\
\text { body fluids and an imbalance in fluids and electrolytes. }\end{array}$ \\
\hline Kikyo & Qi deficiency pattern & $\begin{array}{l}\text { A pattern characterized by decreased vitality, fatigue, weakness, and appetite } \\
\text { loss. It may be explained by deficiency of energy, such as exhausted state, } \\
\text { which is almost always accompanied by deficiency of the upper abdominal } \\
\text { region. }\end{array}$ \\
\hline Kiutsu/Kitai & Qi stagnation pattern & $\begin{array}{l}\text { A pattern characterized by feeling of obstruction at the level of throat, feeling } \\
\text { of ear tube obstruction, abdominal distension due to intestinal gas retention, } \\
\text { depressive state, and intractable pain. It may be explained by functional } \\
\text { discommunication leading to gas retention in bowels, mental depression, or } \\
\text { other manifestations. }\end{array}$ \\
\hline Geshou no kyo & Deficiency in lower energizer pattern & $\begin{array}{l}\text { A pattern characterized by dysfunctions of the urogenital system. It may be } \\
\text { explained by weakness in the lower part of the body. }\end{array}$ \\
\hline
\end{tabular}

(iv) Frequency of stools: although the academic society has no clear-cut standard criteria for this parameter, a stool frequency of once every 3 days or longer was considered as "constipation."

Furthermore, subjective symptoms found in the women and men were analyzed. In women, hie is easy to occur due to decreasing blood levels of estrogen, progesterone [13]. According to the National Livelihood Survey in Japan 1998 by the Ministry of Health and Welfare, women also become aware of hie hands and feet, increased rapidly from around the time of postmenopause over the age of 55. Therefore, we thought that the properties of hie are different in the presence or absence of menstruation. After the overall analysis was performed, the findings in postmenopausal female patients and in women who menstruated were compared (Table 1). The analysis procedure was as follows.

(1) A two-branch decision tree was constructed using CART to classify the data on the basis of the existence or absence of hie.

(2) Based on each decision tree, we performed a knowledge extraction on the following items:

(A) significance test of the presence or absence of hie in each population after divergence;

(B) Kampo-based interpretation of each branch (Table 2); 


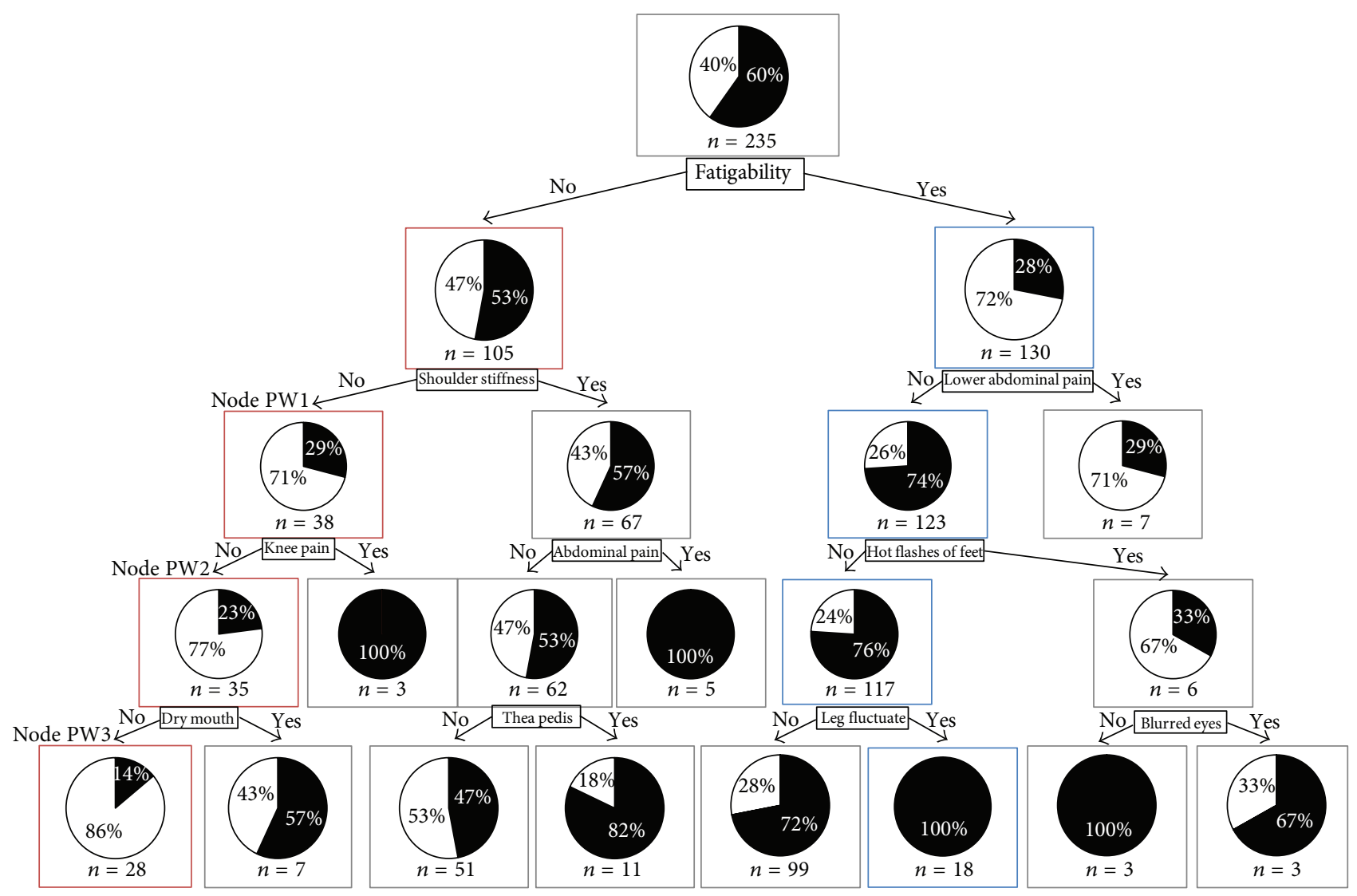

Hie (+)

$\square$ Hie (-)

FIGURE 1: CART of postmenopausal women by hie related symptoms and findings. The root node of Figure 1 indicates that there were 235 total observations $(n=235)$, and the black part of the pie chart means $60 \%$ of " $n$," who have had an episode of hie (hie(+)). And the white part of the pie chart means $40 \%$ of " $n$," who have not had an episode of hie (hie(-)). Each node below the root node has the same meaning.

(C) test of the significance of biases in the findings of each population after divergence.

\section{(3) Comparison of findings.}

CART is a data mining method that allows the classification and regression of samples with multiple, measurable features. Our study method utilized the presence or absence of hie as a patient information label and used the patient interview data as feature variables to repeatedly divide the patient population into 2 exclusive groups based on the patient population size (i.e., large or small, if the feature value was a continuous variable); assignment to a specific category (if the feature variable was a categorical variable, e.g., blood pressure); and rate of hie (e.g., high and low rates). This method allowed for statistical learning based on multiple feature variables, as well as the generation of a decision tree. Significant differences in (2) (A) and (2) (C) were examined using Fisher's exact test to compare the original population (or "base population," namely, the population at the highest level, which was the root node of the decision tree) and the populations after the division. We call this type of analysis subanalysis.

\section{Results}

3.1. Postmenopausal Women. First, a decision tree pertaining to the postmenopausal women group was constructed using CART (Figure 1). Fisher's exact test was performed to detect populations (nodes) with significantly larger (or smaller) numbers of patients with hie, as compared to that of the base population (node 0 ). Originally, $60 \%$ of the patients in the base population presented with hie.

3.1.1. Group of Patients with Significantly More Hie. The result showed that a path formed a population with significantly more hie on the right side of the decision tree than on the left side; in Figure 1, the variables surrounded by blue boxes are included in this path. This path included patients who had the following characteristics: fatigability $(+)$, lower abdominal pain $(-)$, hot flashes of feet $(-)$, and leg fluctuate $(-)$; all patients in the resulting group had hie.

3.1.2. Group of Patients with Significantly Less Hie. On the other hand, in Figure 1, on the left side of the decision tree, a path with red-boxed variables gathers a population with a 
significantly less rate of hie. The patients selected by this path had the following characteristics: fatigability $(-)$, shoulder stiffness (-), knee pain (-), and dry mouth (-); only $14 \%$ of the patients in the resulting group had hie.

Next, the significance of the bias in the findings from the nodes with significant differences in hie was examined using Fisher's exact test. None of the nodes in the path with a significantly more rate of hie showed any finding of bias. Conversely, in the path with significantly less hie, we found several findings.

(i) In the node PW1, the number of patients whose excess of pulse are greater than those with deficiency of pulse (excess $>$ deficiency), while in the base population we found that patients with deficiency of pulse are greater than those with excess of pulse (deficiency $>$ excess).

(ii) In the nodes PW1 and PW2, the tenderness points of blood stasis were found to be $13.6 \%$ and $11.4 \%$ which were approximately $18 \%$ and $20 \%$ lower than that of the result found in the base population (31.5\%), respectively.

(iii) In the node PW3, the number of patients whose humidity of tongue is dry is almost equal to those with normal humidity of tongue and is greater than those with wet (dry = normal > wet), while in the base population we found that patients with normal humidity of tongue are greater than those with dry and dry is much greater than wet (normal $>$ dry $\gg$ wet).

(iv) In the node PW3, the number of patients whose size of tongue are normal is greater than those with enlarge size of tongue and enlarge is greater than thin (normal $>$ enlarge $>$ thin). On the other hand, in the base population we found that patients with normal size of tongue are much greater than enlarge size of tongue and enlarge is greater than thin (normal $\gg$ enlarge $>$ thin).

(v) In the node PW3, the tenderness point of blood stasis was found to be $10.7 \%$, which was $20 \%$ less than that of the result found in the base population.

We summarized the results of subanalysis in Table 3.

3.2. Women with Menstruation. Second, decision tree pertaining to the women with menstruation was constructed (Figure 2). Paths with significantly more (or less) rates of hie were searched through comparison with the base population (node 0) using Fisher's exact test. Originally, 72\% of the patients in the base population presented with hie. Although we described 2 paths with significantly less rates of hie, they were counted as one single path because most of their nodes were common to both.

3.2.1. Group of Patients with Significantly More Hie. In Figure 2, on the right side of the decision tree, we found 2 paths indicated by the blue-boxed variables. These paths comprised a population with significantly more rates of hie. The patients selected by one path had leg swelling $(+)$, and $85 \%$ of the patients in the resulting group had hie. The other
TABLE 3: Summary of subanalysis: findings and $P$ value.

\begin{tabular}{|c|c|c|c|}
\hline & & Findings & $P$ value \\
\hline \multirow{15}{*}{ Node } & \multirow{2}{*}{ PW1 } & Tenderness point of blood stasis & 0.080 \\
\hline & & Excess of pulse & 0.094 \\
\hline & \multirow[t]{2}{*}{ PW2 } & Tenderness point of blood stasis & 0.065 \\
\hline & & Tenderness point of blood stasis & 0.075 \\
\hline & \multirow[t]{2}{*}{ PW3 } & Humidity of tongue & 0.097 \\
\hline & & Size of tongue & 0.044 \\
\hline & W1 & $\mathrm{BMI}<18.5$ & 0.084 \\
\hline & W2 & Blood pressure & 0.016 \\
\hline & W3 & Excess of abdominal strength & 0.062 \\
\hline & W4 & Epigastric tightness and resistance & 0.064 \\
\hline & M1 & Abnormal complexion & 0.012 \\
\hline & M2 & Floating pulse & 0.013 \\
\hline & M3 & Floating pulse & 0.023 \\
\hline & \multirow{2}{*}{ M4 } & Floating pulse & 0.023 \\
\hline & & Epigastric stiffness & 0.013 \\
\hline
\end{tabular}

path had leg swelling (-) and knee pain (+), and all patients in the resulting group had hie.

3.2.2. Group of Patients with Significantly Less Hie. In Figure 2, on the left side of the decision tree, we found a path indicated by the connections of red-boxed nodes (more strictly there are 2 paths, but almost all nodes are shared and we consider the connection of red-boxed nodes as one path). The patients in the terminal nodes (nodes W3 and W4) had leg swelling (-), knee pain (-), abdominal pain $(-)$, and sneezing $(-)$ or $(+)$ and this path formed a population with significantly less rates of hie (63\% of the patients in the node W3 had hie and $44 \%$ of the patients in the node W4 had hie).

The bias of the findings in each node was examined using Fisher's exact test. In the nodes with a significantly more hie rate, we found the following.

(i) From BMI of the patients in the node W1, the percentage of the patients with thin body types was significantly more than that in the base population $(P=0.084)$.

(ii) In the node $\mathrm{W} 2$, there are no patients with hypotension or normal blood pressure, while in the base population they are approximately $22 \%$ for hypotension and $10 \%$ for normal blood pressure. Also, in the base population, while the number of patients with high blood pressure is greater than that of the patients with high-normal, in the node W2 they are switched.

In the path with less rates of hie, namely, in terms of abdominal strength, the following group was determined.

(i) In the node $\mathrm{W} 3$, excess of abdominal strength accounted for $2.7 \%$ of the cases, which was weakly significantly less than that of the observed result of $4.9 \%$ in the base population $(P=0.062)$.

(ii) In the node $\mathrm{W} 4$, the presence of epigastric tightness and resistance accounted for $0 \%$, which was weakly significantly less than that of the observed result of $9.6 \%$ in the base population $(P=0.064)$. 


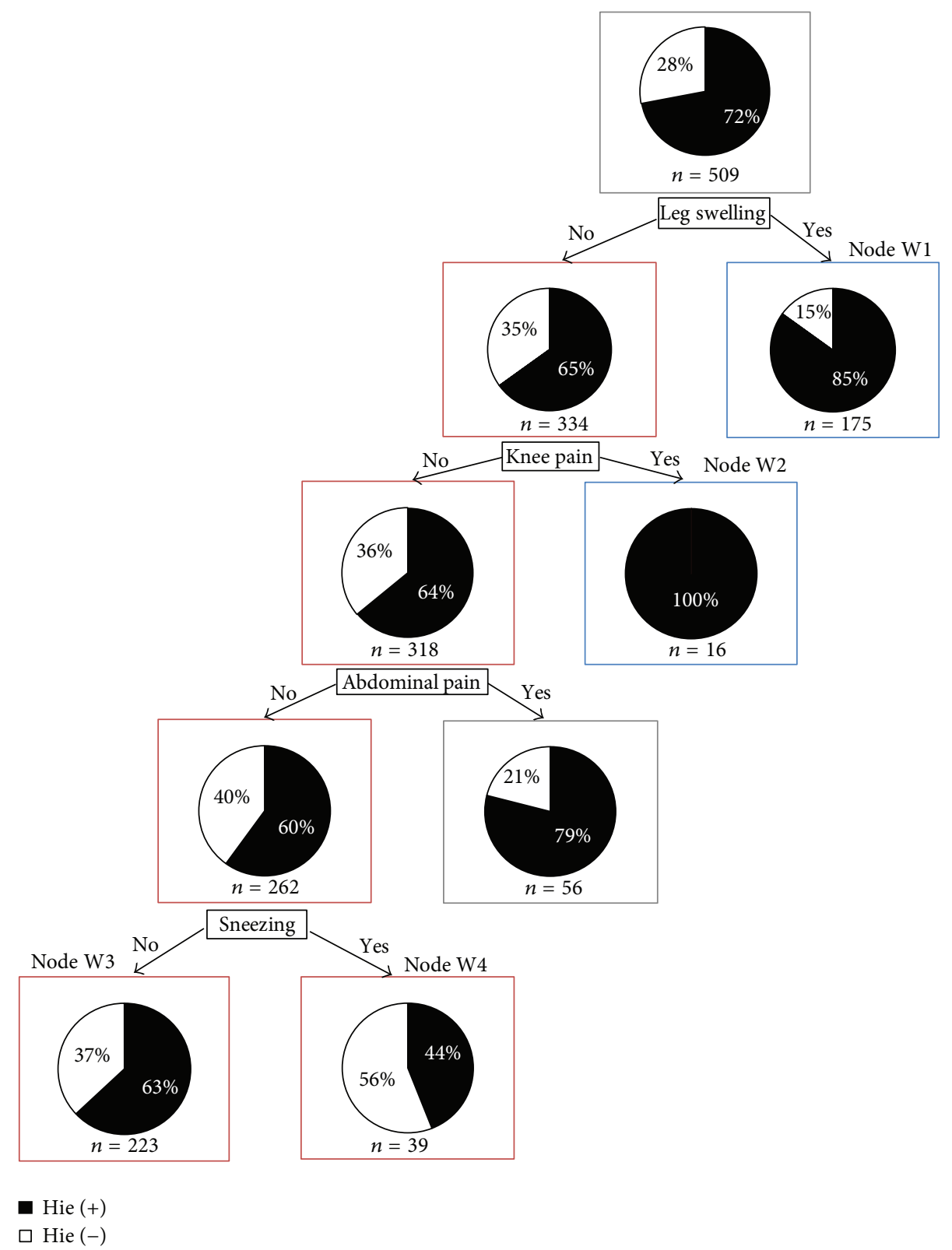

Figure 2: CART of women with menstruation by hie related symptoms and findings. The root node of Figure 2 indicates that there were 509 total observations $(n=509)$, and the black part of the pie chart means $72 \%$ of " $n$," who have had an episode of hie (hie $(+))$. And the white part of the pie chart means $28 \%$ of " $n$," who have not had an episode of hie (hie(-)). Each node below the root node has the same meaning.

3.3. Men. Finally, a decision tree pertaining to hie in men was constructed (Figure 3). Paths that had significant differences in hie were searched using Fisher's exact test and by comparing this with the base population (node 0 ). The findings showed 2 paths with significantly more hie rates and one path with significantly less hie rates.

3.3.1. Group of Patients with Significantly More Hie. In Figure 3, on the right side of the decision tree, we found 2 paths indicated by the connection of blue-boxed nodes. These paths formed a population with significantly more rates of hie. All patients in the terminal node of the 1st path, which is characterized by insomnia (+), leg weakness (+), and excess saliva $(-)$, had hie and in the terminal node of the 2 nd path, with insomnia $(+)$, leg weakness $(-)$, oversensitivity to heat $(+)$, and neck stiffness $(+), 92 \%$ of the patients had hie.

3.3.2. Group of Patients with Significantly Less Hie. In Figure 3, on the left side of the decision tree, we found a path indicated by the red-boxed nodes. This path formed a population with significantly less rates of hie. The patients in the terminal node of this path had insomnia $(-)$, shoulder pain (-), postnasal drip (-), and abdominal pain (-), and only $11 \%$ of patients in the terminal node had hie. 


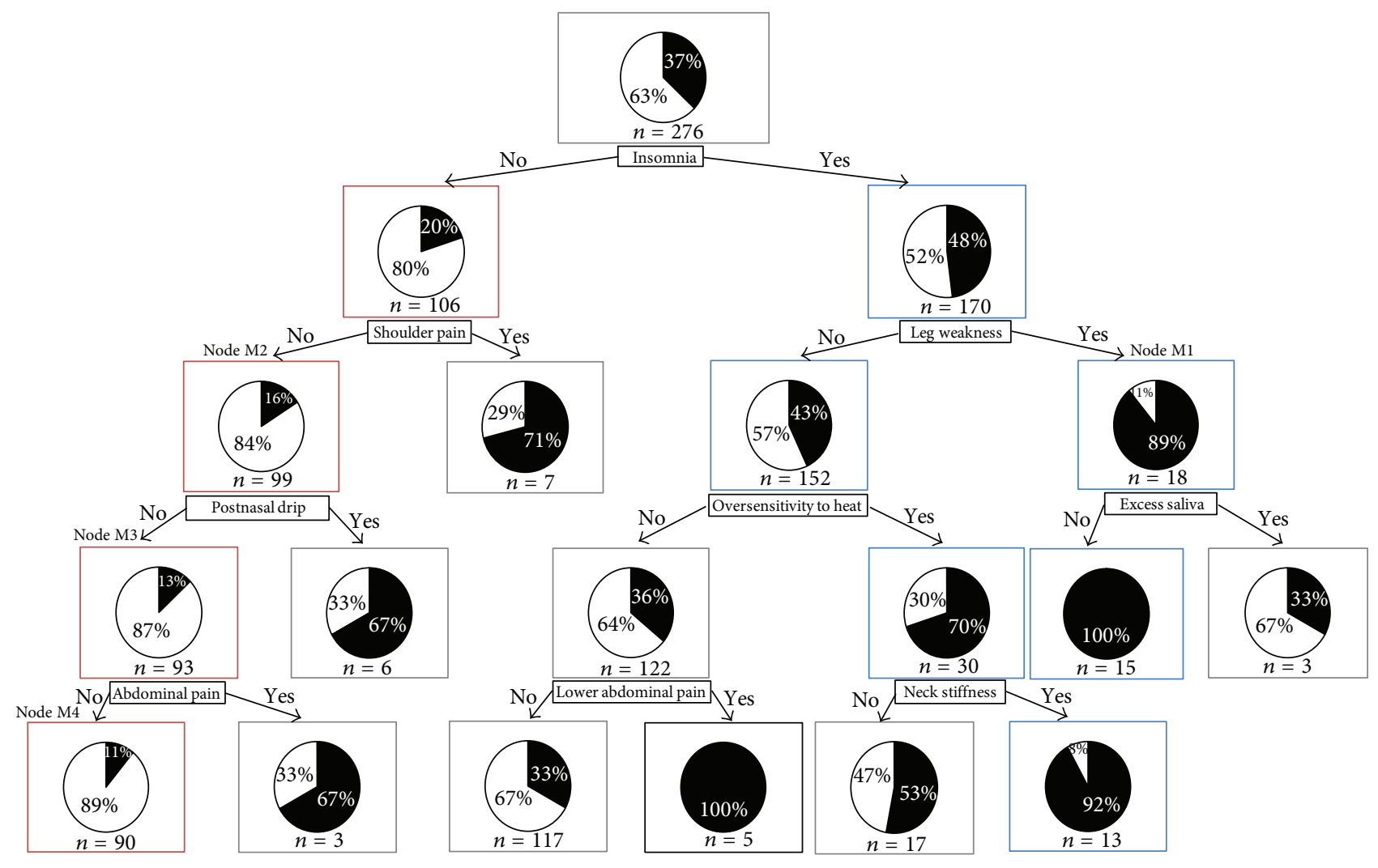

Hie $(+)$

$\square$ Hie (-)

FIGURE 3: CART of men by hie related symptoms and findings. The root node of Figure 3 indicates that there were 276 total observations $(n=276)$, and the black part of the pie chart means $37 \%$ of " $n$," who have had an episode of hie (hie $(+))$. And the white part of the pie chart means $63 \%$ of " $n$," who have not had an episode of hie (hie(-)). Each node below the root node has the same meaning.

Next, bias in the findings in each node was examined using Fisher's exact test. The results can be summarized as follows.

(i) In the 1st path described above, in which the rates of hie were high, namely, in the node M1, abnormal complexion accounted for $46.2 \%$ of the cases, which was significantly more than that of the observed result of $15.2 \%$ in the base population $(P=0.012)$.

(ii) The findings in the 2 nd path showed no significant bias.

(iii) In the path with less hie shown by the red-boxed nodes, we found the following about pulse depth. In the nodes M2, M3, and M4, a floating pulse in these groups accounted for $0 \%$, which was significantly less than that of the observed result of $11.6 \%$ in the base population $(P=0.013$ for node $\mathrm{M} 2, P=0.023$ for node M3, and $P=0.023$ for node M4).

(iv) In the node M4, the presence of epigastric stiffness accounted for $0 \%$, which was significantly less than that of the observed result of $0.72 \%$ in the base population $(P=0.013)$.

\section{Discussions}

In the past, treatment with Kampo medicine was performed based on experience; however, today, medical treatments are based on data. Nevertheless, hie has not yet been studied in a large number of patients.

Previous studies on hie have focused on comparing single variables that may have influenced the development of subjective symptoms among healthy subjects and diagnosed patients [14]. This current study serves as the first effort to analyze hie in a large number of patients using multivariate data mining and statistical analysis methods and to highlight its properties on the basis of strongly related symptoms and their combinations. The results of this study suggested that the symptoms strongly related to hie were different among the groups of postmenopausal women, women with menstruation, and men and that the percentages of hie were different depending on the symptom combinations. By interpreting these symptoms from the perspective of Kampo medicine, common and differing traits for hie in each group were elucidated.

With respect to the findings pertaining to the postmenopausal women with hie, from the Kampo medicine 
perspective, the previously mentioned results suggested that the blood stasis pattern and the Qi deficiency pattern were important in the presence or absence of hie after menopause.

From the perspective of Kampo medicine, the abovementioned results pertaining to the presence or absence of hie in women with a normal menstrual cycle suggested that a blood stasis and water retention pattern played an important role in the development of the hie condition. In the nodes with a large number of patients with hie, the findings were characterized by the unexpected fact that both hypotension and normal blood pressure accounted for $0 \%$, despite the initial expectation for a high percentage.

As for the summary of the findings pertaining to male patients with hie, the results suggest that from the perspective of Kampo medicine a Qi stagnation pattern, a deficiency of lower energizer pattern, and a blood stasis pattern influenced the development of the hie condition.

Interestingly, "oversensitivity to heat $(+)$ " was included despite the fact that the node was comprised of a large number of patients with hie. Hie in this node could probably be attributable to autonomic imbalance.

From this rationale, it became clear that hie and a blood stasis pattern are both concepts of Kampo medicine and are closely related. Most prescriptions used to cure hie actually treat a blood stasis pattern as well. Moreover, hie is closely related to the peripheral circulation disorder. From the viewpoint of the peripheral circulatory failure, the pathology of hie is estimated to be related to the following: a decrease in cardiac output, functional and organic changes in the vessel wall [15], and changes in blood fluidity [16, 17], as well as changes in platelet function, coagulation, and fibrinolysis [18]. From the viewpoint of Western medicine, attempts to clarify the factors involved in a blood stasis pattern have been made.

At our Kampo clinic, our actual experience has shown marked differences between women who menstruate and postmenopausal women, as well as between men and women. Thus, our results statistically confirm what Kampo doctors had already collected during their long clinical experience with respect to the concept hie. The differences in the hie characteristics in each group can be considered to be influenced by differences in the hormonal environment and age-related changes and gender, as well as other factors.

Our research group will conduct further studies using a larger number of patients, include symptoms other than hie, and involve a time-course analysis of the efficacy of the prescribed treatment. We hope this study will contribute to creating more transparency and evidence in the field of traditional Japanese Kampo medicine.

\section{Conclusion}

By using the method of data mining, our study helped to statistically clarify that the characteristics of the concept hie are different among postmenopausal women, menstruating women, and men. Thus, the findings suggested from experience could be confirmed. The results from this study may also support physicians in selecting the appropriate Kampo prescription.

\section{Conflict of Interests}

The authors declare that there is no conflict of interests regarding the publication of this paper.

\section{Acknowledgments}

The authors are most grateful to the editor and the reviewers for the helpful comments on their paper. This work was supported by Grant-in-Aid for Research on Propulsion Study of Clinical Research from the Ministry of Health, Labour and Welfare.

\section{References}

[1] K. Terasawa, "Kampoigaku ni okeru "Hiesho" no ninshiki to sono Chiryo," Shoyakugaku Zasshi, vol. 41, pp. 85-96, 1987 (Japanese).

[2] K. Kushima, "Iwayuru Hieshoni tuite," Sanfujinka no Jissai, vol. 5, pp. 603-608, 1956 (Japanese).

[3] Y. Kimura, "Konenki-shougai to Shuuso," Sanfujinka Chiryo, vol. 76, pp. 136-143, 1998 (Japanese).

[4] M. Suzuki, Y. Hirano, K. Watanabe et al., "Effect of crude drugs preparations for female coldness and related complaints," The Journal of Japan Mibyou System Association, vol. 14, no. 2, pp. 319-322, 2008 (Japanese).

[5] H. Kawagoe, K. Takahashi, A. Kawashima, and T. Ishikawa, "Fact-finding of coldness-fundamental data and frequency of coldness according to diseases," Shindan to Chiryo, vol. 91, pp. 2293-2296, 2003 (Japanese).

[6] T. Murata, "Kampo therapy for coldness-how to diagnose shou and case study," Gendaishuppan Planning, 1993 (Japanese).

[7] H. J. C. M. van de Wal, P. F. F. Wijn, H. J. J. van Lier, and S. H. Skotnicki, "The effectiveness of ketanserin in patients with primary Raynaud's phenomenon. A randomized, double blind, placebo controlled study," International Angiology, vol. 6, no. 3, pp. 313-322, 1987.

[8] C. Franssen, H. Wollersheim, A. de Haan, and T. Thien, "The influence of different beta-blocking drugs on the peripheral circulation in Raynaud's phenomenon and in hypertension," Journal of Clinical Pharmacology, vol. 32, no. 7, pp. 652-659, 1992.

[9] S. B. Wilson, P. E. Jennings, and J. J. F. Belch, "Detection of microvascular impairment in type I diabetics by laser Doppler flowmetry," Clinical Physiology, vol. 12, no. 2, pp. 195-208, 1992.

[10] N. C. Abbot, J. S. Beck, P. D. Samson, C. R. Butlin, P. J. Bennett, and J. M. Grange, "Cold fingers in leprosy," International Journal of Leprosy and Other Mycobacterial Diseases, vol. 60, no. 4, pp. 580-586, 1992.

[11] H. A. J. M. Kurvers, M. J. H. M. Jacobs, R. J. Beuk et al., “The influence of local skin heating and reactive hyperaemia on skin blood flow abnormalities in patients with reflex sympathetic dystrophy (RSD)," European Journal of Clinical Investigation, vol. 25, no. 5, pp. 346-352, 1995.

[12] Y. Gepshtein, G. A. Plotnikoff, and K. Watanabe, "Kampo in women's health: Japan's traditional approach to premenstrual symptoms," Journal of Alternative and Complementary Medicine, vol. 14, no. 4, pp. 427-435, 2008.

[13] S. Kosha, T. Douchi, and Y. Nagata, "Chuukonen Jyosei to Futeishuuso Koureisha no Hie to Hoteri," Sanfujinka Chiryo, vol. 87, pp. 275-279, 2003 (Japanese). 
[14] T. Hanme, H. Koike, and A. Kawashima, "Is coldness cause of various disease," Chiryo Zoukango, vol. 88, pp. 890-892, 2006 (Japanese).

[15] S. I, K. Toriizuka, and S. Tei, "Oketsu to KouKessen ChuuYaku Kenkyu no Ayumi," Kampo to Saishin Chiryo, vol. 2, pp. 310-316, 1993 (Japanese).

[16] H. Abe, M. Oda, T. Takemura, and S. Arichi, "Wakanyaku to Ketsueki Rheology," in Proceedings of the Wakan-Yaku Symposium, vol. 16, pp. 123-125, 1983 (Japanese).

[17] M. Oda, H. Abe, and S. Arichi, "Sekkekkyu Henkeino ni taisuru Keishi-bukuryo-gan no Sayo," Wakan Iyakugaku Kaishi, vol. 1, pp. 243-248, 1984 (Japanese).

[18] K. Terasawa, K. Toriizuka, M. Bandou, A. Imadaya, and H. Tosa, "Effects of medicinal plants on the metabolism of platelet arachidonic acid, studies on "Oketsu" syndrome, platelet aggregation and changes in malondialdehyde values," Journal of Medical and Pharmaceutical Society for WAKAN-YAKU, vol. 2, pp. 310-316, 1985 (Japanese). 


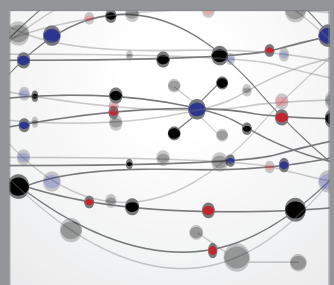

The Scientific World Journal
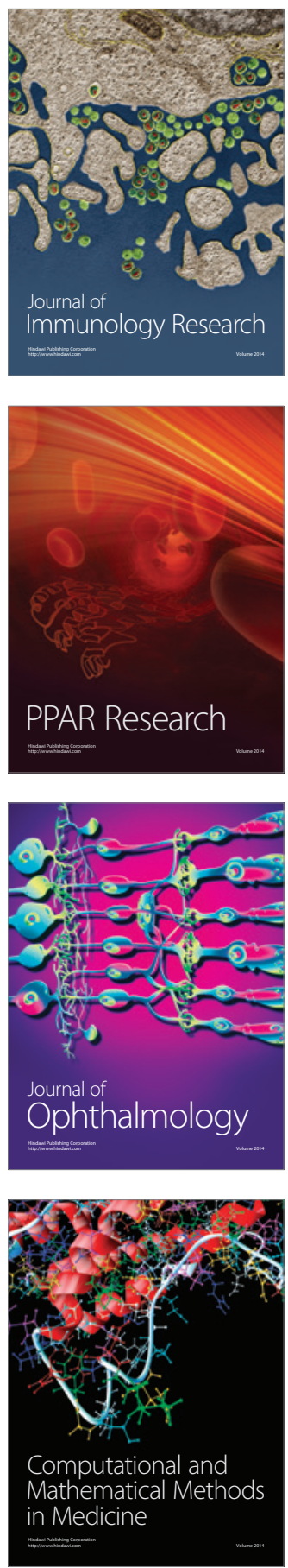

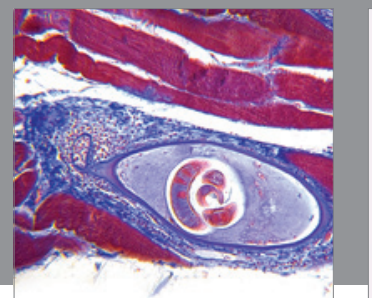

Gastroenterology

Research and Practice
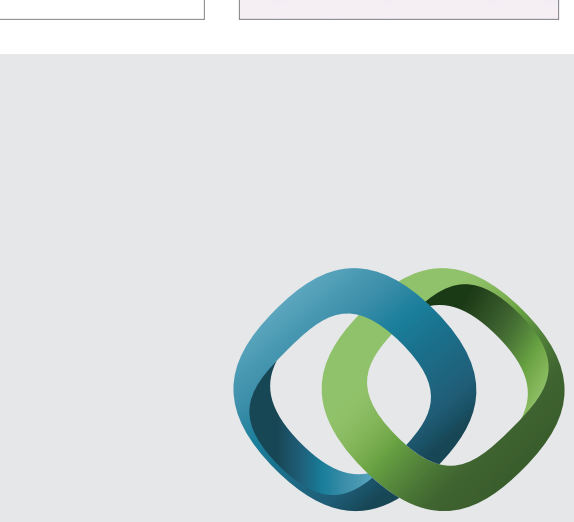

\section{Hindawi}

Submit your manuscripts at

http://www.hindawi.com
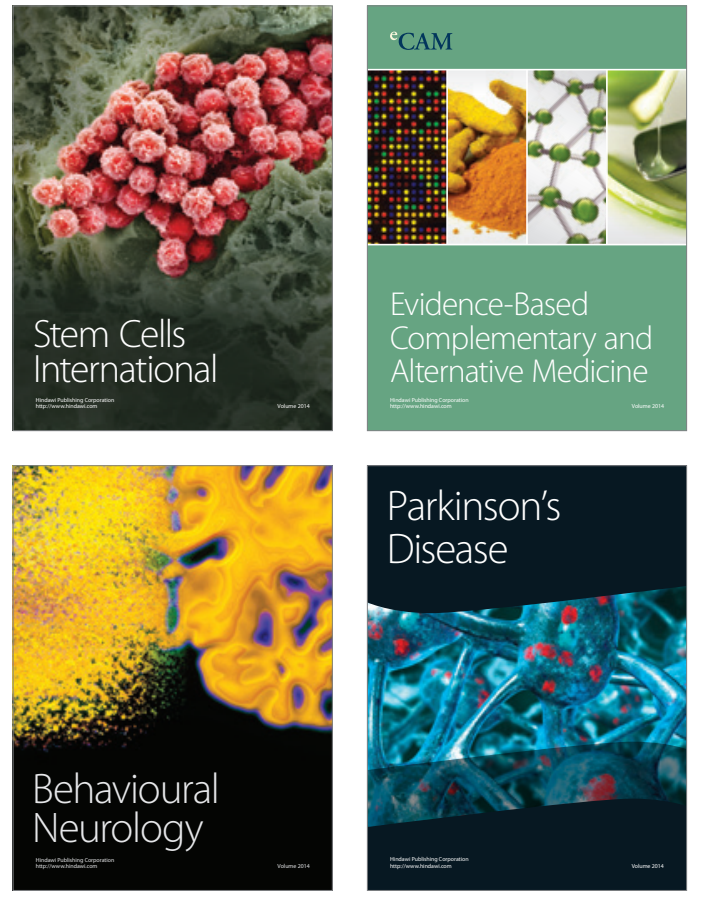
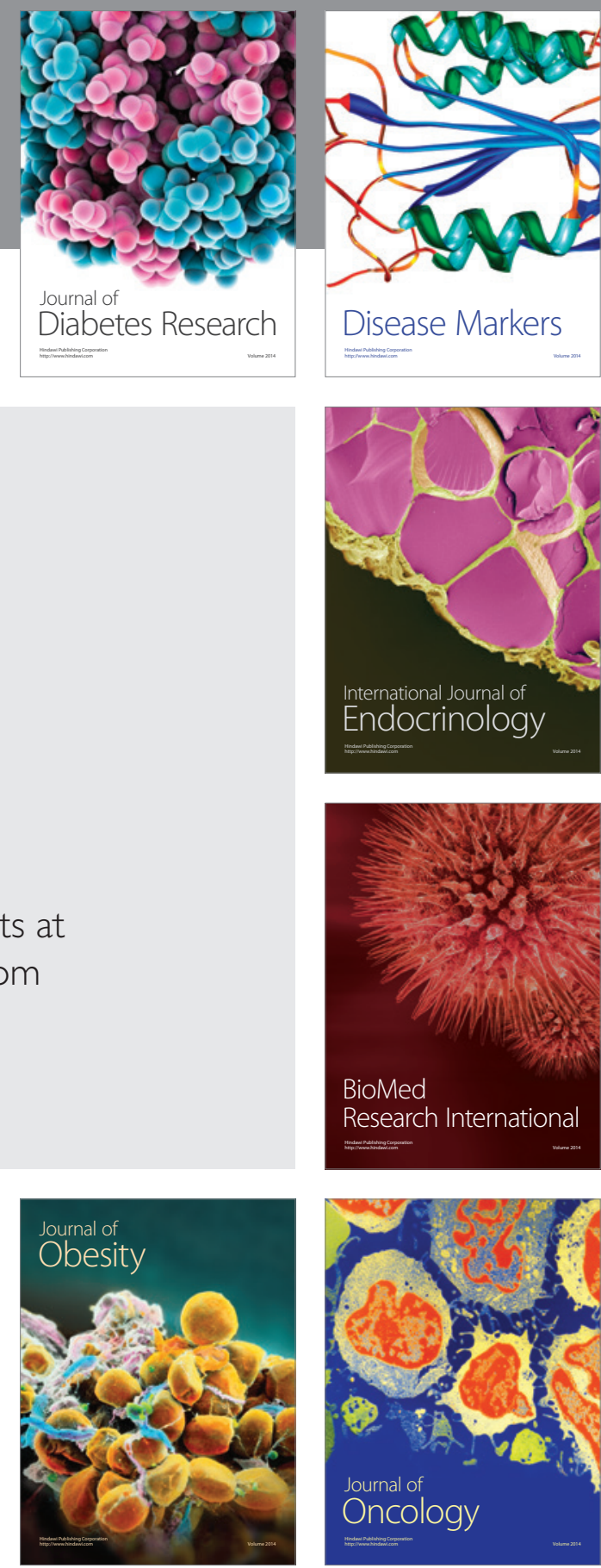

Disease Markers
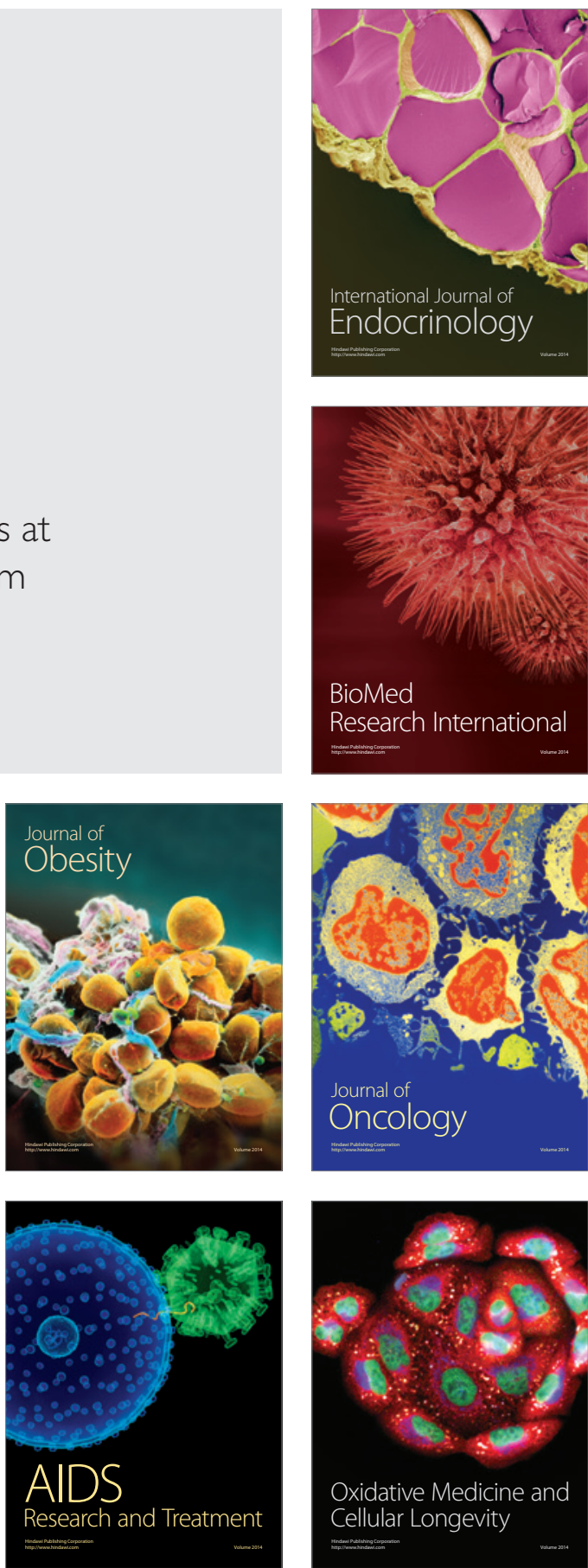